\title{
Meta
}

Journal des traducteurs

Translators' Journal

\section{GRAN, Laura and John DODDS (Eds.) (1989) : The Theoretical and Practical Aspects of Teaching Conference Interpretation, Udine, Campanotto Editore, 278 p.}

\section{Daniel Gile}

Volume 35, numéro 4, décembre 1990

URI : https://id.erudit.org/iderudit/002903ar

DOI : https://doi.org/10.7202/002903ar

Aller au sommaire du numéro

Éditeur(s)

Les Presses de l'Université de Montréal

ISSN

0026-0452 (imprimé)

1492-1421 (numérique)

Découvrir la revue

Citer ce compte rendu

Gile, D. (1990). Compte rendu de [GRAN, Laura and John DODDS (Eds.) (1989) :

The Theoretical and Practical Aspects of Teaching Conference Interpretation,

Udine, Campanotto Editore, 278 p.] Meta, 35(4), 782-783.

https://doi.org/10.7202/002903ar d'utilisation que vous pouvez consulter en ligne.

https://apropos.erudit.org/fr/usagers/politique-dutilisation/ 
GRAN, Laura and John DODDS (Eds.) (1989): The Theoretical and Practical Aspects of Teaching Conference Interpretation, Udine, Campanotto Editore, $278 \mathrm{p}$.

Du 27 au 29 novembre 1986 s'est tenu à Trieste un colloque sur les aspects théoriques et pratiques de l'enseignement de l'interprétation de conférence à l'initiative de l'École de traduction et d'interprétation de l'université de Trieste. Les actes de ce stimulant colloque ont (enfin) été publiés (Laura Gran and John Dodds (eds) (1989): The Theoretical and Practical Aspects of Teaching Conference Interpretation, Udine, Campanotto Editore, 278 pages. On notera en passant qu'à la suite de ce colloque, l'école de Trieste a lancé The Interpreter's Newsletter, le premier (et unique) périodique consacré à l'interprétation de conférence sous l'angle de la théorie et de la recherche. Le deuxième numéro de ce périodique, très riche en contenu, vient de paraître.

Le livre reproduit les communications et les débats du colloque (en anglais et en français). Ses deux caractéristiques les plus marquantes nous semblent être la quantité et la diversité de l'information qu'il présente. À cet égard, il est certainement, et de loin, supérieur à toutes les autres publications sur l'interprétation.

Fort sur le fond, le livre présente toutefois des faiblesses dans la forme: des coquilles et fautes assez nombreuses, des omissions dans les débats qui suivent les communications. D'autre part, alors que certaines interventions dans les débats ont été traduites ou modifiées, d'autres ont été laissées telles quelles alors qu'elles relevaient manifestement de la langue parlée; reproduites sans révision, elles donnent l'impression d'une grande maladresse des intervenants concernés et sont en partie difficiles à comprendre. Ces défauts, ainsi d'ailleurs que des erreurs dans les fonctions et titres des intervenants, auraient pu être évités si les auteurs avaient pu relire les épreuves euxmêmes.

Le livre s'articule en sections: la théorie et ses applications, aspects neurophysiologiques et psycholinguistiques, tests d'aptitude, typologie et analyse des textes, l'enseignement (techniques, méthodologies et cours spéciaux).

Dans la première section, on trouve notamment une analyse critique très pertinente de la recherche en interprétation par Catherine Stenzl, ainsi qu'un tour d'horizon plus général des travaux de recherche et de théorisation par Daniel Gile.

La section neurophysiologique et psycholinguistique nous semble un peu ardue pour le non-spécialiste. En outre, elle se cantonne au niveau général du langage et ne présente pas de résultats suffisamment orientés vers l'interprétation. Sa présence témoigne toutefois d'un certain intérêt des neurophysiologues à l'égard de l'interprétation et a son importance ne serait-ce que par cet aspect.

On notera aussi dans cette section la communication «Recall and Recognition among Conference Interpreters» de Sylvie Lambert qui fait état d'un travail expérimental de type classique, le seul dans le livre. Le sujet est très intéressant en soi: S. Lambert cherche à recueillir des données sur la profondeur de l'analyse du discours par l'interprète en comparant la rétention du discours après écoute, shadowing (répétition avec décalage), simultanée et consécutive. Résultats : l'écoute semble accompagnée de l'analyse la plus poussée (car elle est associée aux scores de rétention les plus élevés); elle est suivie par la consécutive et la simultanée, puis le shadowing. Intéressant, dans la mesure où l'écoute est sensée être passive et devrait donc en principe être accompagnée d'une analyse moins profonde que l'interprétation. Ces résultats semblent confirmer l'intérêt de la consécutive dans l'enseignement de l'interprétation, dans la mesure où il en ressort que la consécutive favorise l'analyse plus que la simultanée. Toutefois, on peut se poser des questions méthodologiques: dans quelle mesure la rétention réflète-t-elle vraiment la profondeur de l'analyse? Dans quelle mesure l'emploi du test t bilatéral se 
justifie-t-il avec un échantillon non aléatoire de huit professionnels et de huit étudiants seulement?

Dans la troisième section, David et Margareta Bowen font une description détaillée, exemples à l'appui, de leur procédure de sélection des candidats à l'école de Georgetown.

Dans la cinquième section, on notera la présence de deux représentants de l'Europe de l'Est : Ilona Zalka, de Budapest, et Jurij Denissenko, de Moscou, qui défend la thèse du travail vers le $\mathrm{B}$ et affirme que les interprètes occidentaux tendent à faire du transcodage, alors que les interprètes soviétiques ont une démarche plus sémantico-communicationnelle (!). On y trouve également des descriptions détaillées de techniques d'enseignement mises en œuvre à Monterey (Wilhelm Weber et Ine Mary Van Dam), et un intéressant système raisonné pour la prise de notes en consécutive présenté par Sergio Allioni.

Dans la sixième section, on remarquera entre autres une analyse détaillée des stages de formation pour interprètes confirmés par Jennifer Mackintosh, et une interrogation iconoclaste de Michael Francis sur l'opportunité de l'enseignement de la consécutive qui donne à réfléchir (la communication de Sylvie Lambert y répond en partie).

Le livre se distingue donc de la majorité des publications sur l'interprétation par le fait qu'il présente des informations, beaucoup plus qu'il ne formule des théories ou qu'il ne prend position sur des questions de fond.

On y trouve toutefois aussi des prises de position, intéressantes surtout parce que certaines tranchent avec d'autres opinions, plus présentes dans les publications parce que plus souvent réitérées (mais toujours par le même petit groupe d'auteurs).

Il est frappant de noter que plusieurs intervenants (L. Cosson, G. Ilg, J. Mackintosh, J. Neff, E. Nowak-Lehmann, E. Spiller, A. Bossatra, W. Weber) parlent d'automatisation dans l'interprétation, un mot jusqu'ici tabou et qui a d'ailleurs provoqué un vif échange lors du colloque (non reproduit dans les actes). Pour la plupart des intervenants, il signifie essentiellement la réduction des besoins en capacité de traitement là où la chose est possible afin d'en laisser davantage pour les opérations où elle est vraiment nécessaire.

Tout aussi significative d'une évolution dans les mentalités, l'évocation de l'apprentissage des langues en cours de formation (initiale ou continue) par D. et M. Bowen, L. Cosson, J. Mackintosh et F. Prete, qui tranche avec certaines affirmations catégoriques faites ailleurs.

Enfin, la spécificité de l'interprétation par langues, catégoriquement réfutée par certains, est mentionnée par J. Altman, J. Denissenko, C. Stenzl et Yen Ping Cheng.

Au total, le livre présente 37 communications. La politique de sélection des organisateurs du colloque semble avoir été très libérale, ce qui a permis à des jeunes et à des intervenants peu connus de s'exprimer. L'inconvénient de cette politique est un niveau très inégal des contributions. En l'occurrence, nous pensons que cette politique libérale était justifiée, dans la mesure où le Colloque de Trieste a donné l'occasion à des intervenants d'exprimer des opinions que l'on entend peu en général. On notera tout de même que les tendances de l'ESIT, opposées à plusieurs idées fortes formulées lors du colloque, y sont peu représentées faute de représentants (C. Thiéry était le seul ambassadeur de l'école de Paris sur place).

En résumé, en raison de la quantité et de la diversité des informations qu'il contient ainsi que des idées en général non publiées auxquelles il donne l'occasion de figurer en bonne place dans la littérature, ce livre nous semble mériter d'être lu par tous les collègues s'intéressant à l'enseignement de l'interprétation, et plus généralement aux théories et à la recherche sur l'interprétation. Italie.

Il peut être commandé chez Campanotto Editore, Via Michelini 1, 33100 Udine, 